\title{
THE SOLUTION SPACES FOR INTEGRAL EQUATIONS OF THE SCATTERING THEORY ${ }^{1}$
}

\author{
BY ERGUN AR
}

\author{
Communicated by L. Cesari, March 21, 1967
}

The integral equations expressing the scalar wave functions for the exterior region of a smooth and bounded scatterer in terms of the potential Green's function have recently been found [2], [3]. In the following note we discuss the solutions for these equations.

1. The statement of the problem. Let $B$ denote the boundary of a smooth, closed and bounded surface in $E^{3}$, and $V$ the exterior of $B$. Erect a spherical polar coordinate system with origin interior to $B$; and denote by $r$ a point $(r, \theta, \phi)$ in $V$ and by $\boldsymbol{r}_{B}$ a point $\left(r_{B}, \theta_{B}, \phi_{B}\right)$ on $B$. Further let $v$ be a scalar wave function for the exterior $V$, i.e.,

(a) $v(r)$ is of class $C^{2}, r \in \bar{V}=V \cup B$,

(b) $\left(\nabla^{2}+k^{2}\right) v(\boldsymbol{r})=0, \boldsymbol{r} \in \bar{V} ; k$, the wave number, is assumed to be complex,

(c) $r(\partial v / \partial r-i k v)=o(1), r \rightarrow \infty$, uniformly in $\theta$ and $\phi$.

Then, we obtain the equation

$$
\omega=K_{1} \omega+u_{1}^{(0)}
$$

for the Dirichlet problem, and the equation

$$
\omega=\left(K_{1}+K_{2}\right) \omega+u_{2}^{(0)}
$$

for the Neumann problem; where

$$
\begin{gathered}
\omega=\exp (-i k r) v, \\
\omega \rightarrow K_{1} \omega=-2 i k \int_{V} d v_{1} \frac{G_{0}\left(r, r_{1}\right)}{r_{1}} \frac{\partial}{\partial r}\left[r_{1} \omega\left(r_{1}\right)\right], \\
\omega \rightarrow K_{2} \omega=i k \int_{B} d \sigma_{B} G_{0}\left(r, r_{B}\right) \hat{n} \circ \hat{\boldsymbol{r}}_{B} \omega\left(\boldsymbol{r}_{B}\right), \\
u_{1}^{(0)}=\int_{B} d \sigma_{B} \omega\left(\boldsymbol{r}_{B}\right) \frac{\partial}{\partial n} G_{0}\left(r, \boldsymbol{r}_{B}\right), \\
u_{2}^{(0)}=-\int_{B} d \sigma_{B} G_{0}\left(r, \boldsymbol{r}_{B}\right) \exp \left(-i k r_{B}\right) \frac{\partial \omega\left(\boldsymbol{r}_{B}\right)}{\partial n} .
\end{gathered}
$$

1 This work is supported by NSF Grant GP-6140 at the Radiation Laboratory, The University of Michigan. 
In the above equations $d v$ is the volume element, $d \sigma$ the surface element, $\nabla^{2}$ the Laplacian expressed in the coordinates $(r, \theta, \phi) ; \hat{n}$ and $\hat{\boldsymbol{r}}_{B}$ are unit vectors in the normal and radial directions respectively, and $\partial / \partial n$ denotes the normal derivative. $G_{0}$ denotes the potential Dirichlet Green's function when it is used in (D), and the potential Neumann Green's function when it is used in $(\mathrm{N})$.

We assume that the surface $B$ can be described by an equation $r_{B}=g(\theta, \phi)$. For the Dirichlet problem it is sufficient to assume that $g \in C^{1}(B)$ while for the Neumann problem an additional restriction is needed; namely, that $\hat{n} \circ \boldsymbol{r}$ is uniformly Hölder continuous on $B$.

2. Construction of a solution space. Let $W$ be the set of functions defined on $\bar{V}$ such that

(a) $\omega \in C^{2}(V), \omega \in C^{1}(\bar{V})$,

(b) $\omega$ is analytic in the complex $z=1 / r$ plane having the expansion

$$
\omega=\sum_{n=0}^{\infty} f_{n}(\theta, \phi) z^{n+1} \quad|z| \leqq \frac{1}{\delta},
$$

$\delta=c+\epsilon, \epsilon>0$, and $c$ is the radius of the smallest sphere containing the surface $B$,

(c) $f_{n}(\theta, \phi)=\sum_{m=n}^{\infty} Y_{m}(\theta, \phi)$, where $Y_{m}$ is an $m$ th-order spherical harmonic, i.e.,

$$
Y_{m}(\theta, \phi)=\sum_{l=-m}^{m} A_{l m} P_{m}^{l}(\cos \theta) \exp (i l \phi) .
$$

We define the following norm on $W$,

$$
\|\omega\|=\max _{\boldsymbol{r} \in \overline{\bar{V}}}|\omega(\boldsymbol{r})|+\max _{|z| \leqq \delta}|\omega(\theta, \phi, 1 / z)|,
$$

for all $0 \leqq \theta \leqq \pi, 0 \leqq \phi \leqq 2 \pi$.

Then in $\{W,\|\cdot\|\}$ the solutions for the equations (D) and (N) are found in the usual manner. Specifically, turning our attention to the Dirichlet problem, we let

$$
\omega^{(N)}=\sum_{n=0}^{N} K_{1}^{n} u_{1}^{(0)}
$$

and show that (a) $u_{1}^{(0)} \in W$, (b) $K_{1}$ maps $W$ into itself, (c) $\left\|K_{1}\right\|<1$ for sufficiently small $|k|$, (d) $\omega \in W$, and (e) $\omega^{(n)} \rightarrow \omega$ as $N \rightarrow \infty$ in norm (7). Thus, using the relation (1) we obtain the solution of the scalar Helmholtz equation for the exterior region $V$. The procedure, with more complicated details, is essentially the same for the Neumann problem. 
3. Further results. (a) If we define the norm as

(8) $\|\omega(\boldsymbol{r})\|=\max _{\boldsymbol{r} \in \overline{\bar{V}}}|\omega(\boldsymbol{r})|+\max _{\boldsymbol{r} \in \overline{\bar{V}}}\left|D^{\prime} \omega(\boldsymbol{r})\right|+\max _{\boldsymbol{z} \leqq \delta}|\omega(r, \theta, 1 / z)|$,

for all $0 \leqq \theta \leqq \pi, 0 \leqq \phi \leqq 2 \pi$, where $D^{\prime}$ denotes any one of the first derivatives with respect to $\theta, \phi$ or $r$, then with a slight change (6) becomes a complete space. Then the operators in (D) and (N) are contraction operators (for small enough $|k|$ ) in norm (8), and therefore, by a Banach fixed point theorem these equations are uniquely solvable. This fact and the relation (1) provide new existence proofs for the Dirichlet as well as the Neumann problems for the exterior Helmholtz equations.

(b) One of the surfaces for which the Helmholtz equation is nonseparable is a spindle (a football shaped object). However, the Laplace equation is partially separable (and therefore solvable) in the exterior region of this body. By means of the above technique the scalar wave function for the Dirichlet problem has been found for this case [1], though the result remains formal due to the edges on the spindle's surface. All the details will be given elsewhere.

4. Acknowledgment. The author is indebted to R. E. Kleinman and G. W. Hedstrom for helpful discussions.

\section{REFERENCES}

1. Ergun Ar, On the low frequency acoustical scattering of a plane wave from a soft spindle at nose-on incidence, Quart. Appl. Math. (to appear).

2. Ergun $\mathrm{Ar}$ and R. E. Kleinman, The exterior Neumann problem for the threedimensional Helmholtz equation, Arch. Rational Mech. Anal. 23 (1966), 218-236.

3. R. E. Kleinman, The Dirichlet problem for the Helmholtz equation, Arch. Rational Mech. Anal. 18 (1965), 218-236.

The University of Michigan 